\title{
Outcomes of transcatheter aortic valve replacement for pure native aortic regurgitation with the use of newer- vs. early- generation devices
}

\author{
Wei-Hsian Yin ${ }^{1,2}$, Yung-Tsai Lee ${ }^{1,3}$, Tien-Ping Tsao ${ }^{1,4}$, Kuo-Chen Lee ${ }^{1,4}$, Ming-Chon Hsiung $^{1}$, Jeng Wei $^{1,4}$ \\ ${ }^{1}$ Heart Center, Cheng Hsin General Hospital, Taipei; ${ }^{2}$ Faculty of Medicine, School of Medicine, National Yang Ming Chiao Tung University, Taipei; \\ ${ }^{3}$ Institute of Microbiology and Immunology, School of Medicine, National Yang Ming Chiao Tung University, Taipei; ${ }^{4}$ Faculty of Medicine, National \\ Defense Medical Center, Taipei \\ Contributions: (I) Conception and design: WH Yin, J Wei; (II) Administrative support: WH Yin, J Wei; (III) Provision of study materials or patients: \\ All authors; (IV) Collection and assembly of data: WH Yin, YT Lee, J Wei; (V) Data analysis and interpretation: WH Yin, YT Lee, J Wei; (VI) \\ Manuscript writing: All authors; (VII) Final approval of manuscript: All authors. \\ Correspondence to: Jeng Wei. Heart Center, Cheng Hsin General Hospital, No. 45, Cheng Hsin St, Beitou, Taipei. Email: jengwei@mac.com.
}

Background: Accumulated experience and advances in device technology have led to the increasing off-label use of transcatheter aortic valve replacement (TAVR) for pure native aortic valve regurgitation (PNAR). This study aimed to evaluate the procedural and long-term outcomes of using newer-generation transcatheter heart valves (THVs) versus early-generation self-expanding CoreValve (Medtronic, Minneapolis, USA) to treat PNAR.

Methods: TAVRs were performed with the use of early- $(\mathrm{N}=15)$ and newer-generation $(\mathrm{N}=10)$ THVs in a total of 25 consecutive PNAR patients at an intermediate-to-high risk for surgical aortic valve replacement [mean Society of Thoracic Surgeons (STS) score of 6.8 4.5]. Procedural and clinical outcomes were reported according to the Valve Academic Research Consortium 2 criteria. The primary end-point of the study was all-cause mortality, myocardial infarction (MI), disabling stroke, and readmission due to heart failure.

Results: The device success rate of the newer-generation THVs was significantly higher than that of the early-generation CoreValve $(100 \%$ vs. $33 \%, \mathrm{P}<0.01)$, which was mainly driven by less frequent need for implanting a second THV $(0 \%$ vs. $53 \%, \mathrm{P}<0.01)$. Although the procedural success rates were $100 \%$ for both early- and newer-generation valves, the mean procedure fluoroscopic times which the newer-generation device group required, were significantly shorter $(\mathrm{P}<0.01)$ and the amount of contrast medium used in this group, markedly smaller $(\mathrm{P}<0.01)$, compared to those of the early-generation CoreValve group. During a median follow-up of 14 months, event-free survival was better in patients undergoing TAVR with the newer-generation THVs, although the differences were not statistically significant (log-rank test, $\mathrm{P}=0.137$ ). According to multivariate analysis, a higher baseline STS score and longer intensive care unit stays are independent predictors of adverse outcomes.

Conclusions: Evidently, the treatment of PNAR with TAVR using the newer-generation THVs yielded better procedural outcomes and is a valuable therapeutic option in selective patients.

Keywords: Transcatheter aortic valve replacement (TAVR); pure native aortic regurgitation (AR); transcatheter heart valves (THVs); procedural and clinical outcomes

Submitted Nov 30, 2021. Accepted for publication Jan 07, 2022.

doi: $10.21037 /$ atm-21-6936

View this article at: https://dx.doi.org/10.21037/atm-21-6936 


\section{Introduction}

The use of transcatheter aortic valve replacement (TAVR) for patients with severe aortic stenosis has recently been expanded from patients with high- or intermediate-risk to low-risk patients (1). Nevertheless, the application of TAVR in severe pure native aortic valve regurgitation (PNAR) remains limited (2-11). This is due to the fact that aortic valves with degenerative aortic regurgitation (AR) typically lack annular or leaflet calcifications, which are required for anchoring the transcatheter heart valves (THVs). However, severe PNAR is usually associated with a high mortality rate if left untreated (12-14). For elderly PNAR patients with multiple comorbidities, such as severe left ventricular (LV) dysfunction, previous stroke, gastrointestinal bleeding, etc., the surgical risk is prohibitively high (2). Evidently, for such PNAR patients, there is an unmet need and less invasive treatments such as TAVR are needed.

The Medtronic CoreValve prosthesis (Medtronic Inc., Minneapolis, MN, USA), with its self-expanding property and longer frame, which offers additional anchorage of the valve stent in the $\mathrm{LV}$ outflow tract and ascending aorta, was used in early attempts of TAVR for PNAR (2-6). Unfortunately, the device failure rate was high, mainly driven by a high rate of requiring a second THV and the presence of significant residual paravalvular leakage (PVL) (2-6,9-11). In recent years, newer-generation THVs, such as the Evolut R or Evolut PRO (Medtronic Inc.) selfexpanding valves, fortified with a smaller-caliber delivery system, a fully recapturable platform, and a wider range of annular diameter applicability than the smaller CoreValves; or THVs with specific anchoring designs dedicated for PNAR, such as the JenaValve (JenaValve Technology, Irvine, CA, USA) and J-valve (JieCheng Medical Technology, Suzhou, China), have been introduced, with promising early clinical results (3-11).

Although already in clinical utilization and their early safety and clinical efficacy have been documented, it is unknown if the newer-generation THVs truly improves TAVR long-term outcomes in PNAR patients in realworld practice. In the present study, we aimed to compare the procedural and long-term clinical outcomes of TAVR for PNAR using the early-generation Medtronic CoreValve prosthesis versus the newer-generation THVs in symptomatic PNAR patients. Since the heart team at Cheng Hsin General Hospital is one of the largest and the most experienced in Taiwan, the present study does offer an opportunity to examine how the evolution of THVs affected the clinical outcomes of PNAR patients undergoing TAVR over time in a "real-world" clinical setting of a single large volume center.

We present the following article in accordance with the TREND reporting checklist (available at https://dx.doi. org/10.21037/atm-21-6936).

\section{Methods}

\section{Patient population}

In the present study, a total of 25 consecutive patients from April 2015 to November 2020 were recruited; all of them had severe PNAR. Since the patients were at a high risk for conventional cardiac surgery with sternotomy and cardiopulmonary bypass, they were referred to our TAVR multidisciplinary team. Each patient's clinical presentations, medical history, and family history were recorded during their first visit to the hospital. In our institution, a shared decision-making approach is adopted for all patients considering aortic valve replacement, with implementation of best practices to ensure the incorporation of patient goals and preferences into the final decision. This study was approved by Institutional Review Board of Cheng Hsin General Hospital [No. (523) 104-59-3], and was conducted in accordance with the Declaration of Helsinki (as revised in 2013). The requirement for individual consent for this retrospective analysis was waived.

\section{Choice of device, vascular access, and TAVR procedures}

The choice of device was determined by the heart team and based on patients' clinical conditions and anatomies. The TAVR procedure was first performed in Taiwan in 2010. Whereas, the most widely available early valve technologies were the Medtronic CoreValve, Lotus (Boston Scientific, Natick, MA, USA), and Spien XT (Edwards Lifesciences, Irvine, CA, USA), and they were launched in 2012, 2015 and 2016, respectively. Given the very long frame anchors of the CoreValve, which not only holds on to the aortic annulus but also extends up to the supra-coronary aorta, thereby improving its stability, we used CoreValve as the default TAVR device for all PNAR before 2017 when surgery was not an option (i.e., for "no-option" patients). The newer-generation self-expandable Evolut $\mathrm{R}$ was introduced in Taiwan in 2017. Since it can be recaptured and repositioned, the prosthesis has theoretically become more easily maneuvered. Hence, the Evolut R valve was 
used in seven PNAR patients requiring TAVR from 2018 to 2019.

The J-valve is a PNAR-dedicated newer-generation TAVR device. It has a unique system consisting of three U-shape graspers, which not only facilitate intuitive selfpositioning implantation, enabling both axial as well as radial fixation by enwrapping the native valve leaflets, but also achieve fine positioning and active fixation to the leaflets. The initial experiences reported in the literature were promising (9). Therefore in 2020, three J-valves were approved by the Taiwan Food \& Drug Administration for compassionate use in three critically ill PNAR patients.

All of the THV implantations were performed in our hybrid theater, and all patients were treated under general anesthesia. Moreover, the default strategy for all of our patients was the transfemoral (TF) approach, although the J-valve could only be implanted via the transapical approach. TF TAVR was conducted using percutaneous closure devices or after the surgical cut-down of the femoral artery in cases of vessel calcifications or severe obesity. When a transcatheter approach for PNAR had been deemed to be appropriate, we paid our attention to the procedural technique to overcome challenges in dealing with severe PNAR. Next, self-expanding valves oversizing by at least $15-20 \%$ were selected to accommodate the more expansile aortic regurgitant valve, due to the lack of calcium. We also used rapid pacing at 130-140 beats per minute for valve deployment to allow stable anchoring. To improve visualization and reduce the risk of malposition, we sometimes placed two pigtail catheters in the aortic root to give a clearly defined fluoroscopic coplanar annular view.

As for the transapical approach for J-valve implantation, anterolateral mini-thoracotomy was performed in the fifth or sixth intercostal space to obtain direct access to the $\mathrm{LV}$ apex, which was best determined by the preoperative chest computed tomography (CT) scan. The J-valve delivery system was inserted in the left ventricle and across the aortic valve along the guidewire. The process of implantation was monitored by both fluoroscopy and intra-operative transesophageal echocardiography, and was completed in two stages. The first stage was positioning the claspers in the aortic root. After the claspers had been seated in the aortic sinuses, the second stage was performed by lowering and deploying the prosthesis into the aortic annulus. As soon as the prosthesis had been deployed and released, the delivery system was removed. This step-by-step implantation has already been demonstrated in a previous publication (9). The apical sutures were then tied after the removal of the guidewire, and the incision was closed in a routine fashion.

All of the patients were referred to an intensive care unit post-TAVR and were monitored there for at least 1 day. Meanwhile, heart rate monitoring was not dis-continued until discharge. Platelet inhibition was performed by the application of aspirin $100 \mathrm{mg}$ per day in all patients. Moreover, an additional dose of $75 \mathrm{mg}$ of clopidogrel was administered post-procedurally for 3 months in most cases. However, patients with an indication for anticoagulant therapy received clopidogrel and warfarin, or a direct oral anticoagulant without aspirin.

\section{Follow-up and data collection}

Echocardiography and clinical follow-up were performed before and after the operation. The heart-valve team was in charge of the patients' follow-up after their discharges, which included telephone interviews and office visits. Data were prospectively collected and entered into our "heart valve replacement database".

Prediction of patient mortality after TAVR was calculated using the Society of Thoracic Surgeons predicted risk of mortality (STS-PROM). Echocardiographic studies performed at baseline and after TAVR were evaluated according to the criteria of the American Society of Echocardiography (15).

\section{Definitions}

Severe PNAR was defined as severe aortic valve regurgitation with an aortic valve area $>1.5 \mathrm{~cm}^{2}$, but without calcification on the aortic leaflets or annulus, as determined by transthoracic echocardiography and aortography. Aortic valve calcification was assessed using CT. All patients included in this study were defined as grade I (no calcification). Patients with concomitant moderate AS (mean gradient $\geq 20 \mathrm{mmHg}$ ), AR due to degeneration of a transcatheter or surgical bioprosthesis, and patients with acute $\mathrm{AR}$ or active endocarditis were excluded.

According to the Valve Academic Research Consortium-2 consensus document (16), device success was defined as follows: (I) absence of procedural mortality, (II) correct positioning of a single prosthetic heart valve into the proper anatomical location, and (III) intended performance of the prosthetic heart valve without prosthesis-patient mismatch (mean aortic valve gradient $<20 \mathrm{mmHg}$ or peak velocity $<3 \mathrm{~m} / \mathrm{s}$ ), and no moderate nor severe prosthetic valve 
regurgitation. Procedural success was defined as a successful deployment of the TAVR device and retrieval of the delivery system without mortality, conversion to surgical aortic valve replacement, nor myocardial infarction (MI).

The main endpoints of this study were the major cardiac and cerebral adverse events (MACCE) in terms of all-cause mortality, major stroke, $\mathrm{MI}$, and readmission due to heart failure during follow-up (15).

\section{Statistical analysis}

Data were transferred from the database to the Statistical Program for Social Sciences program (version 18.0 for Windows, SPSS Inc., Chicago, IL, USA). Univariate comparisons of demographic, procedural and outcome parameters between these two groups were conducted. Continuous variables were expressed as mean \pm standard deviation and were compared using the Student's $t$-test or the Wilcoxon rank sum test. Categorical variables were presented as percent frequency and were compared by the Pearson's chi-square test or the Fisher's exact test. Logistic regression analysis was used to identify the predictors of device success in the study patients.

As for the survival analysis, the TAVR patients were divided into two groups, depending on whether or not MACCE occurred during follow-up. Univariate comparisons of clinical characteristics and laboratory measurements between the two groups were conducted using appropriate tests. The independent predictors of MACCE in the study patients were determined using multivariate Cox proportional hazards analyses. Variables with a $\mathrm{P}$ value $<0.1$ in the univariate analysis were included in the multivariate model, in addition to the use of early- $v s$. newer-generation valves.

A two-sided $\mathrm{P}<0.05$ was considered statistically significant for all analyses. Statistical analysis was performed using SPSS version 18.0 statistical software.

\section{Results}

\section{Baseline characteristics and echocardiographic measurements of the study patients (Table 1)}

The baseline demographic and clinical characteristics of patients who underwent TAVR with early- vs. newergeneration devices are summarized in Table 1. In general, the two groups were well matched. The incidence of coronary artery disease was higher in patients undergoing TAVR with early- vs. newer-generation devices, but was statistically insignificant ( $47 \%$ vs. $10 \%, \mathrm{P}=0.09)$. Although patients in the CoreValve implantation group were more inclined to have previous stroke, they tended to experience more New York Heart Association (NYHA) functional class III/IV heart failure at presentation and had higher STS-PROM compared to those who underwent newer-generation device implantation, but the differences were not statistically significant. Moreover, the baseline echocardiographic measurements showed markedly lower right ventricular ejection fraction $(54.5 \% \pm 8.1 \%$ vs. $60.8 \% \pm 4.0 \%, \mathrm{P}=0.02)$ and more $\geq$ moderate mitral regurgitation in the early- $v$. newer-generation groups ( $87 \%$ vs. $40 \%, \mathrm{P}=0.03$ ).

\section{Procedural characteristics and immediate results of the study patients}

The technical aspects of the procedure and procedural outcomes are presented in Table 2. The valve sizes ranged from 26 to $31 \mathrm{~mm}, 29 \mathrm{~mm}, 26$ to $34 \mathrm{~mm}$, and 27 to $29 \mathrm{~mm}$ for the CoreValve, Sapien XT, Evolut R, and J-valve, respectively. The mean valve sizes used and valve oversizing in both groups were similar, although more $31 \mathrm{~mm}$ CoreValve were used in the early-generation device group, as the $31 \mathrm{~mm}$ CoreValve was the largest valve available before 2017 . In three patients in the newergeneration device group, we had to implant $34 \mathrm{~mm}$ Evolut R valves. The TAVR procedures were conducted transfemorally for all CoreValves, Evolut R valves, and Sapien XT valves. The three J-valves were implanted via transapical access, and two of them were $29 \mathrm{~mm}$ valves. The final implantation depth below the annulus was notably shallower in patients of the newer-generation valve group, compared to that of the early-generation CoreValve group $(8.5 \pm 3.2$ vs. $14.1 \pm 5.5 \mathrm{~mm}, \mathrm{P}<0.01)$.

Eight patients with CoreValve required implantation of a second valve because of an initial low implantation to left ventricle, resulting in malposition and severe PVL. All of the PVL complicating low deployments of a CoreValve prosthesis were treated by transcatheter insertion ("TAVRin-TAVR") of a second THV. A CoreValve-in-CoreValve procedure was performed in six of the eight patients; significant PVL ( $\geq$ moderate degree) after TAVR procedure was found in two of them (Table 2, Figure $1 A, 1 B$ ). The other two (of the eight) patients with initial low implantation of the CoreValve underwent TAVR with a second Sapien XT prosthesis at the level of the CoreValve waist, and the results in both cases were satisfactory (Table 2, 
Table 1 Baseline characteristics and echocardiographic measurements of the study patients

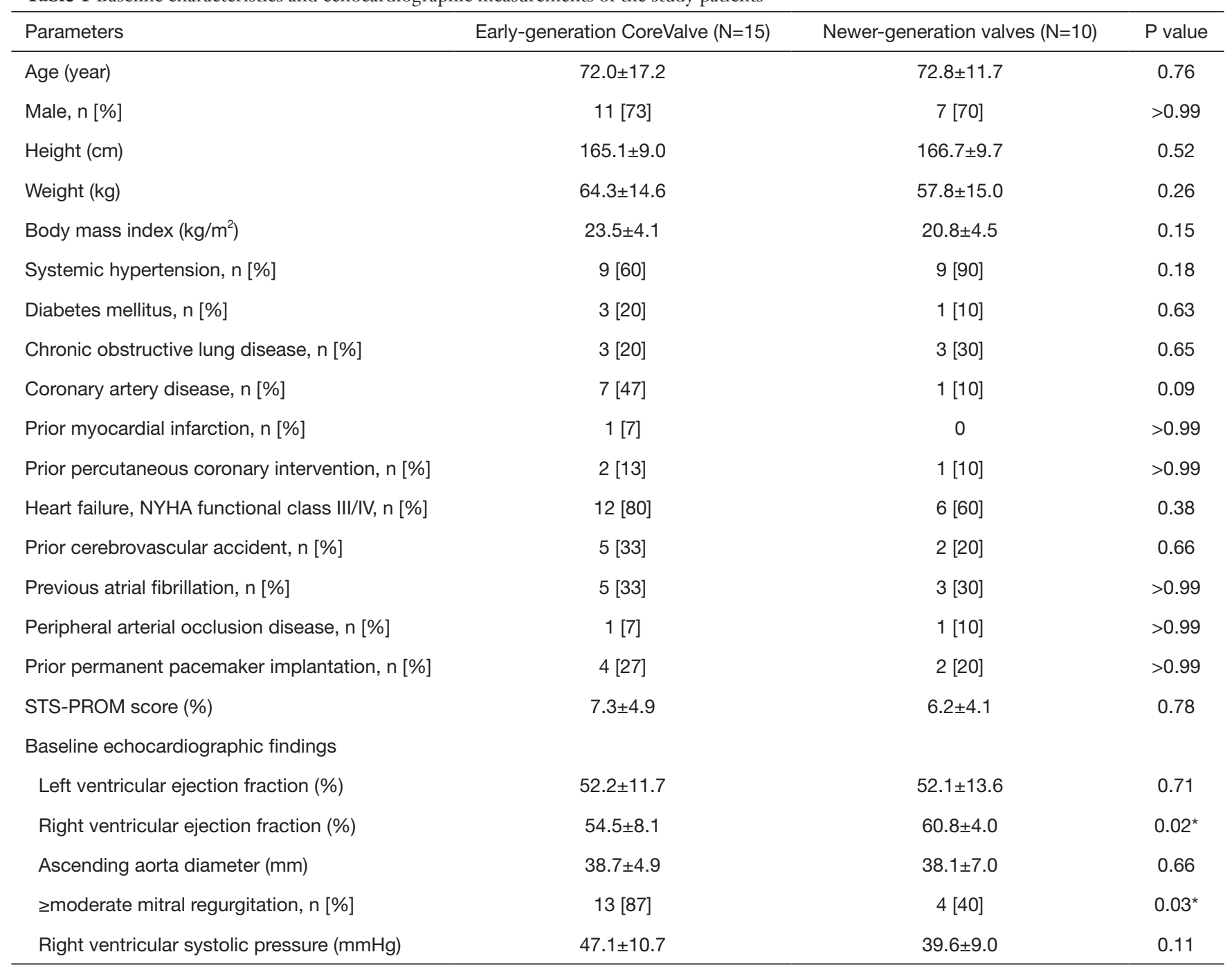

Continuous variables are expressed as mean \pm standard deviation. ${ }^{*}$, significance level, $\mathrm{P}<0.05$. NYHA, New York Heart Association; STSPROM, Society for Thoracic Surgery-probability of mortality score.

Figure 1C,1D). In summary, the device success rate was $33 \%$ only for early-generation CoreValve and $100 \%$ for newergeneration valves, and the latter was significantly higher than the former $(\mathrm{P}<0.01)$. Device failure was mainly driven by the need for a second valve implantation [53\% for earlygeneration CoreValve and $0 \%$ for newer-generation valves $(\mathrm{P}<0.01)$, and to a lesser extent, by residual significant $\mathrm{PVL}$ [13\% for early-generation CoreValve and $0 \%$ for newergeneration valves $(\mathrm{P}=0.50)$ ].

Table 2 also demonstrated that the incidences of major intraoperative complications, which include emergency conversion to open-heart surgery, annular or LV rupture, coronary occlusion, or the need for emergency hemodynamic support, and the rate of new pacemaker implantation for complete heart block, were similar between the two groups. The procedural success rates were $100 \%$ for both; however, the mean procedure and fluoroscopic times spanned significantly longer in the early-generation CoreValve group, compared to those of the newer-generation device group $(37.1 \pm 14.1$ vs. $64.9 \pm 26.4$ minutes, $\mathrm{P}<0.01$ and $19.3 \pm 4.7$ vs. $41.5 \pm$ 13.3 minutes, $\mathrm{P}<0.01$, respectively). Also, the newergeneration TAVR procedures used markedly less contrast versus the early-generation THV implantation $(88.1 \pm 52.0$ vs. $165.1 \pm 69.3 \mathrm{~mL}, \mathrm{P}<0.01)$. The logistic regression analysis showed that the use of newer-generation THVs 
Table 2 Procedural characteristics, immediate results, and clinical outcomes of the study patients

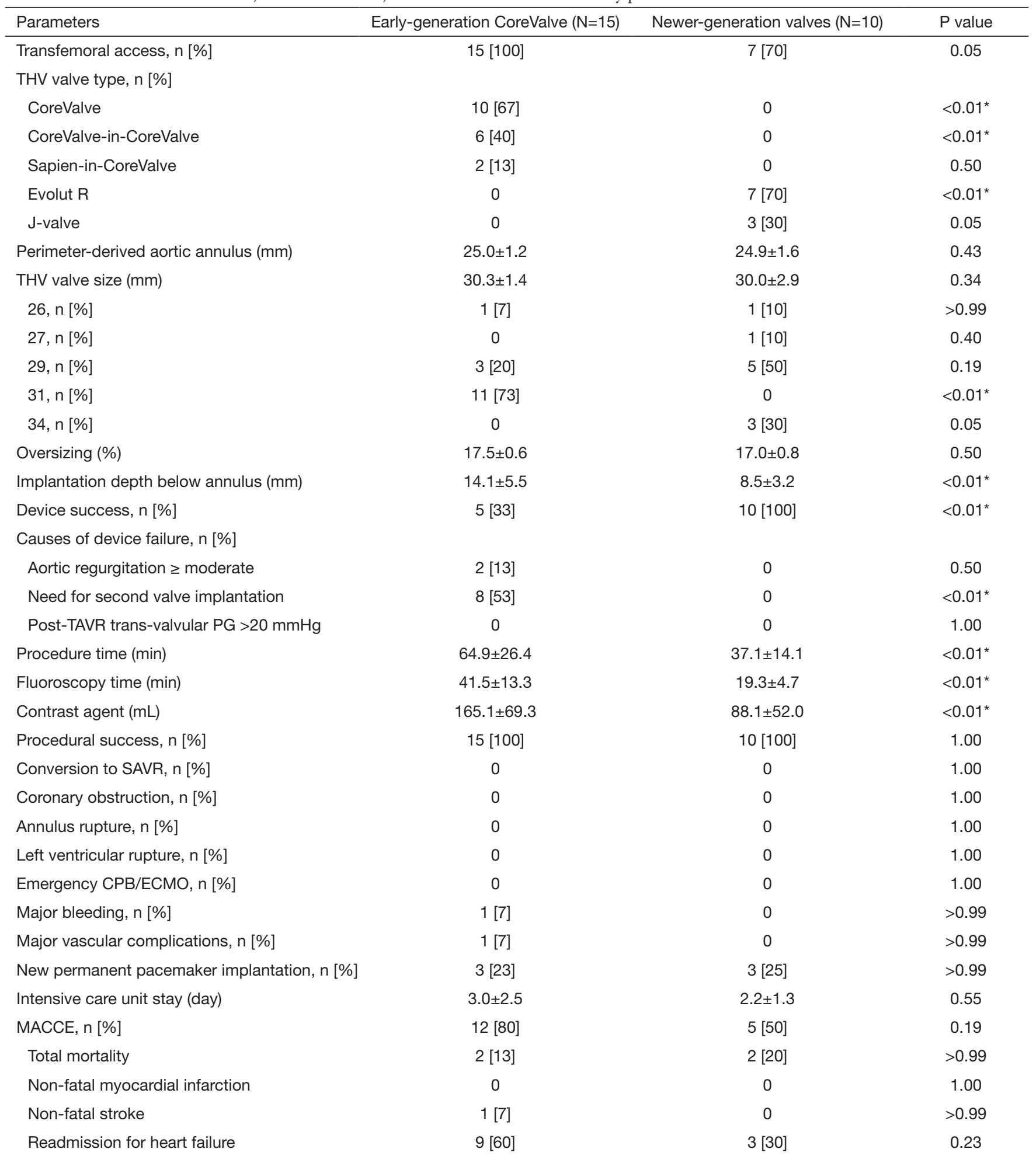

Continuous variables are expressed as mean \pm standard deviation. ${ }^{*}$, significance level, $\mathrm{P}<0.05$. THV, transcatheter heart valve; TAVR, transcatheter aortic valve replacement; PG, pressure gradient; SAVR, surgical aortic valve replacement; CPB, cardio-pulmonary bypass; ECMO, extracorporeal membranous oxygenation; MACCE, major adverse cardiac cerebral events. 

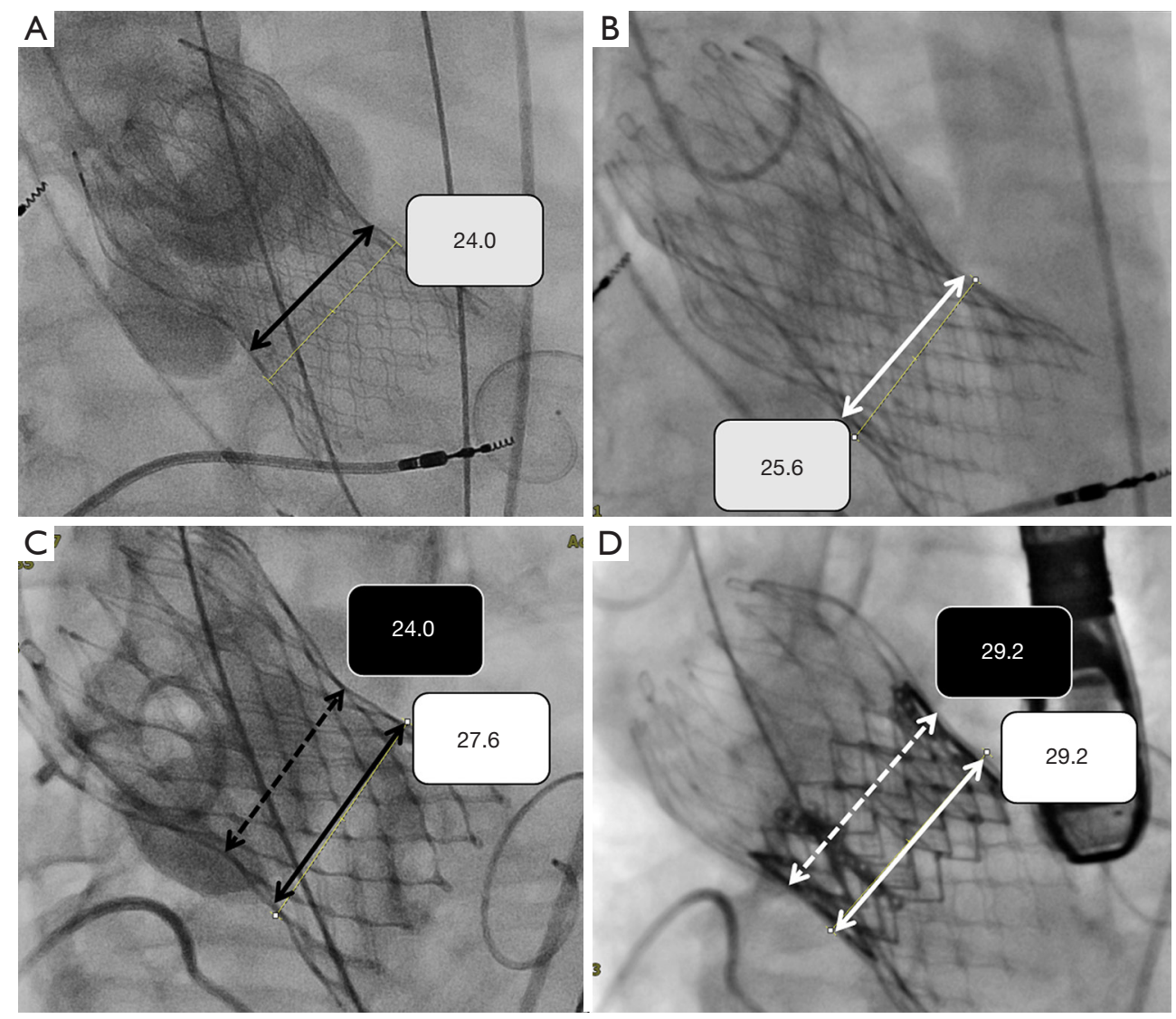

Figure 1 Positioning the CoreValve often proved to be very difficult because of the turbulence due to the pure native aortic regurgitation. The valve may be deployed too deeply into the left ventricle and causes moderate to severe residual paravalvular leakage (PVL). In our series, all of the PVL complicating low deployments of a CoreValve prosthesis were treated by transcatheter insertion ("TAVR-in-TAVR") of a second transcatheter heart valve. (A) In one of the patients needing CoreValve-in-CoreValve implantation, significant residual PVL was evident, owing to a lack of radial strength of the second $31 \mathrm{~mm}$ CoreValve to afford enough stent expansion of the first $31 \mathrm{~mm}$ CoreValve. The diameter of the first CoreValve frame at its waist was $24.0 \mathrm{~mm}$ (black double arrow), occupying the annulus in this case. Implantation of the second CoreValve increased the diameter at the waist from 24.0 to $25.6 \mathrm{~mm}$ (white double arrow), with significant recoil (B). In another patient with low deployment of a $31 \mathrm{~mm}$ CoreValve prosthesis, the diameter of the device at the aortic annulus was $27.6 \mathrm{~mm}$ (black double arrow) and $24.0 \mathrm{~mm}$ at its waist (dashed black double arrow) (C). We implanted a $29 \mathrm{~mm}$ Sapien XT valve in the malpositioned CoreValve in order to eliminate the residual PVL. The final diameters both at the annulus (white double arrow) and the waist (dashed white double arrow) were $29.2 \mathrm{~mm}$ (D).

was the only significant determinant of device success (Table 3).

\section{Clinical outcomes}

The intensive care unit stays were similar between the two groups. During a median follow-up of 14 months, there were no significant differences in all-cause mortality, nonfatal MI, non-fatal stroke, or re-admission due to heart failure. Kaplan-Meier analysis showed that the event-free survival rate of patients who underwent TAVR with newergeneration THVs, was better but not statistically significant (log-rank test, $\mathrm{P}=0.137$ ) (Figure 2).

The TAVR patients were then divided into two groups depending on whether or not MACCE occurred during follow-up. In the multivariate Cox proportional hazards analyses, the independent predictors of MACCE in the study patients were determined using variables such as device types, which were associated with the MACCE in the univariate analysis. Multivariate analysis identified that 
Table 3 Logistic regression analysis to identify the predictors of device success in the study patients

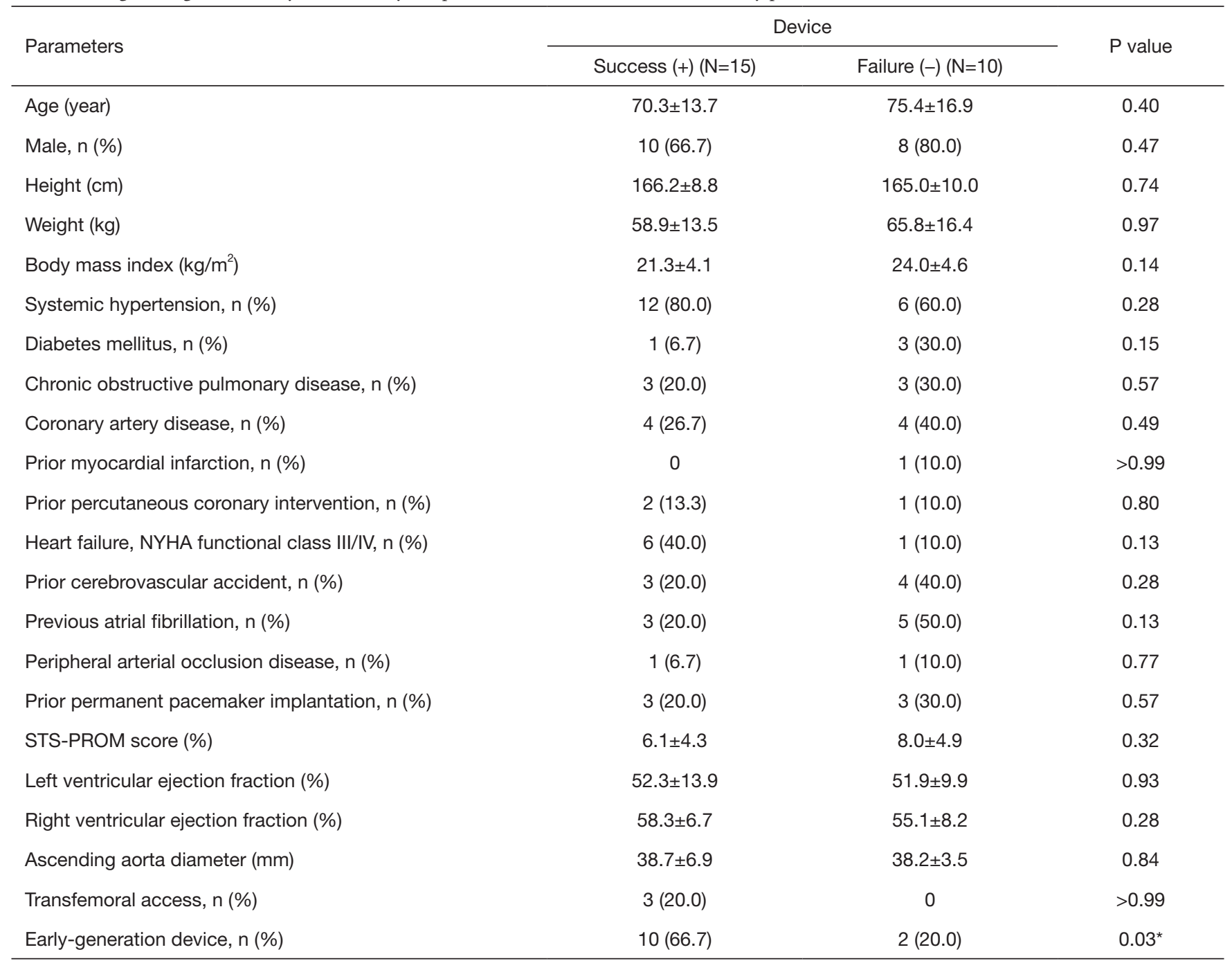

Continuous variables are expressed as mean \pm standard deviation. *, significance level, $\mathrm{P}<0.05$. NYHA, New York Heart Association; STSPROM, Society for Thoracic Surgery-probability of mortality score.

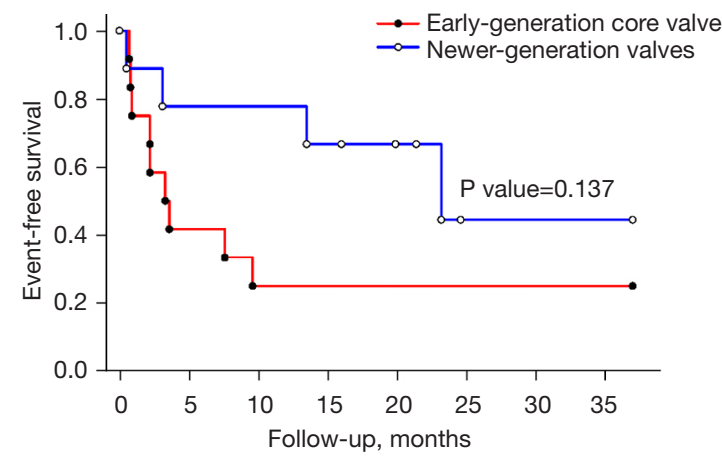

Figure 2 Event-free survival curve of the early- $(\mathrm{N}=15)$ vs. newergeneration $(\mathrm{N}=10)$ devices. the baseline STS-PROM score $(\mathrm{P}<0.01)$ and intensive care unit stays $(\mathrm{P}=0.02)$ were independently associated with the primary end-points of combined all-cause mortality, MI, disabling stroke, and readmission due to heart failure within 48 months (Table 4).

\section{Discussion}

As afore-mentioned, the present study aimed to offer an opportunity to examine how the evolution of THVs and other clinical factors affected the procedural and longterm clinical outcomes of PNAR patients undergoing 
Table 4 Independent prognostic determinants of MACCE by univariate and multivariate analysis

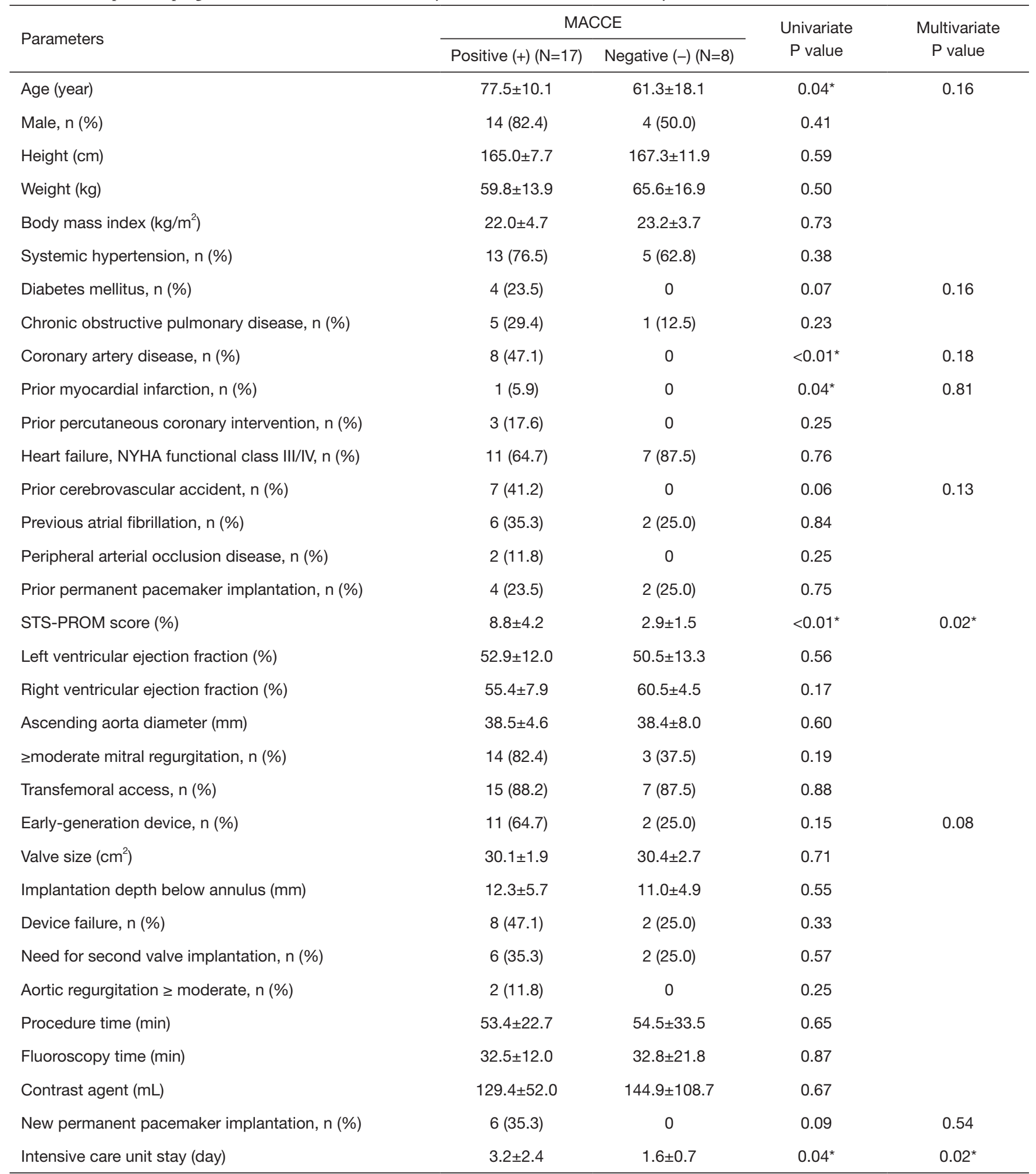

Continuous variables are expressed as mean \pm standard deviation. *, significance level, $\mathrm{P}<0.05$. MACCE, major adverse cardiac and cerebral events; NYHA, New York Association; STS-PROM, Society for Thoracic Surgery-probability of mortality score. 
TAVR over time in a "real-world" clinical setting of a single large volume center. The main findings of our study are as follows. Firstly, the outcomes of off-label use of early-generation CoreValve TAVR device in patients with PNAR have been suboptimal, mainly driven by THV malpositioning and residual PVL. Secondly, PVL complicating low deployment of a CoreValve prosthesis can be eliminated by adopting the transcatheter TAVRin-TAVR approach with a balloon-expandable prosthesis at the level of the CoreValve waist. Thirdly, the newergeneration Evolut R may improve TAVR outcomes, but still has limitations such as new-onset conduction abnormalities. Lastly, the early results of the dedicated J-valve for PNAR are promising, but further research on a larger scale is needed.

TAVR devices were originally designed for use in patients with aortic stenosis, with calcium providing an anchoring point for device deployment. PNAR affects about $13 \%$ of patients suffering from isolated native left-sided valvular heart disease (12). Symptoms related to PNAR tend to appear at a later phase in the course of the disease, when LV dilatation and systolic dysfunction have already developed. Patients with severe PNAR and LV ejection fraction $<30 \%$ have an annual mortality risk of $20 \%$, but only $5 \%$ of them undergo surgical aortic valve replacement for real (12). Left untreated, severe PNAR is associated with a high mortality rate $(13,14)$.

However, the absence of annular and leaflet calcification, much needed for device anchoring and stabilization during deployment, is the main challenge facing interventionists during TAVR for PNAR. In addition, dilatation of the ascending aorta, strong regurgitant jets, and dramatic deformation dynamics of the aortic annulus make device positioning and deployment very difficult. Hence, the prosthesis has a predisposition to embolization or malposition, causing subsequent moderate to severe residual PVL (2-6). Valves with PNAR are more elastic, and thus, can expand to a greater degree during valve deployment. Accordingly, TAVR devices in PNAR are typically oversized to accommodate a greater stretch in the valve. Although both THV under- and over-sizing were associated with an increased risk of THV malpositioning in TAVR for PNAR (6), there is no definitive manufacturer recommendation regarding the degree of oversizing. Previous reports recommended oversizing by at least $15-20 \%(17,18)$, and that for those patients with larger aortic annuli, valves may need to be oversized up to $25 \%$ of the largest annular diameter in systole (3). In our study, the mean annular diameters and valve oversizing were $25.0 \pm 1.2 \mathrm{~mm}$ and $17.5 \% \pm 0.6 \%$, and $24.9 \pm 1.6 \mathrm{~mm}$ and $17.0 \% \pm 0.8 \%$ in the CoreValve and the newer-generation device groups, respectively. However, only the $31 \mathrm{~mm}$ CoreValve was available prior to 2017, when four of the 13 PNAR cases $(31 \%)$ with perimeterderived annular diameters of $26 \mathrm{~mm}$ or more had to undergo $31 \mathrm{~mm}$ CoreValve implantation, with $\leq 16 \%$ oversizing. These patients could have had a $34 \mathrm{~mm}$ Evolut $\mathrm{R}$ valve implanted for TAVR ( $>24 \%$ oversizing) if it were available, just as the three out of 10 patients $(30 \%)$ in similar situations underwent $34 \mathrm{~mm}$ Evolut R implantation between 2018 and 2019. This may explain why the device success rate has improved in our series with the use of the newer-generation Evolut R valves. Judging by our experiences in dealing with PNAR, at least $20 \%$ oversizing is appropriate in patients with large annular diameters.

Residual moderate to severe PVL in TAVR for PNAR is considered a predictive factor for TAVR failure, recurrent heart failure, and mortality (2-6). With the use of newergeneration devices, moderate or severe residual PVL can be markedly reduced (2-6). Our data are consistent with those previous studies. According to our series, malpositioning with too-low implantation of the CoreValve was presented in $8 / 13$ patients $(62 \%)$, resulting in moderate to severe residual PVL and heart failure. The options for treatment in this setting include attempted withdrawal of the malpositioned device by using a snare (19), device occlusion of PVL (20), or modification of the CoreValve waist by inserting a second CoreValve (21). Unfortunately, we discovered that the radial strength of the second CoreValve may not be enough to sustain the required stent expansion, and in two of the six patients requiring CoreValve-inCoreValve implantation, significant residual PVL were still evident (Table 2 and Figure 1A). After reviewing earlier reports, we found one case of aortic stenosis, reported by Wilson et al. in 2014, in which a $29 \mathrm{~mm}$ Sapien XT valve was inserted within a $31 \mathrm{~mm}$ CoreValve to resolve severe PVL in the setting of low deployment of a $31 \mathrm{~mm}$ CoreValve prosthesis. The same report also provided convincing bench testing results to demonstrate the ability of a Sapien XT valve in a CoreValve; that is, it can resist recoil and achieve expansion of the CoreValve waist (22). In our series, we successfully treated two patients with this Sapien-in-CoreValve approach before the retrievable, recapturable Evolut R system was available (Table 2 and Figure $1 B$ ). In spite of the rapid technical improvements and increasing safety of newer-generation self-expanding THV 
implantation, malpositioning remains an issue with TAVR procedures. Under these circumstances, we recommend that transcatheter insertion of a balloon-expandable valve into the first deeply deployed self-expanding prosthesis is feasible and may be a better salvage the procedure in terms of reducing residual PVL $(22,23)$. Furthermore, the increased potential risk of impaired coronary access in patients undergoing TAVR-in-TAVR with two supraannular devices may also be avoided (24).

Despite the fact that newer-generation devices have achieved lower rates of valve malposition, second valve insertion, and significant residual PVL, their clinical results are overall worse than those of the TAVR for aortic stenosis. Actually, the need for permanent pacemaker implantation (PPI) has not been improved with newergeneration self-expanding devices (2-6). The depth at which a THV is implanted in the LV outflow tract has been consistently associated with the requirement for new PPI. A shallower THV implantation is the most straightforward strategy to reduce new PPI rates (25-29). Although our data showed that the final implantation depth below the annulus was significantly shorter in patients using the newer-generation valves $v s$. those who received the earlygeneration CoreValve $(8.5 \pm 3.2$ vs. $14.1 \pm 5.5 \mathrm{~mm}, \mathrm{P}<0.01)$, the incidences of new PPI were similar in both groups in our study (newer-generation valves $v s$. CoreValve $=25 \%$ vs. $23 \%, \mathrm{P}<0.01)$. We considered that a mean implantation depth of $8.5 \pm 3.2 \mathrm{~mm}$ in the newer-generation THV group was still too deep, compared to those studies specifically designed to minimize PPI after TAVR, either by using the MInimizing Depth According to the membranous Septum (MIDAS) approach or cusp overlap technique (27-29). Another possible explanation for the deeper implantation in TAVR for PNAR is the operator's anxiety that a shallow implantation may result in aortic embolization (popup), especially with the use of a larger $34 \mathrm{~mm}$ Evolut R. Therefore, the operators usually implant the valve a little deeper to avoid embolization (30). However, simply a few millimeters of implantation depth are enough to make a drastic difference for PPI rates. Moreover, the higher new PPI rates in TAVR for PNAR with the use of a larger selfexpanding valve may also be attributed to the anchoring mechanism of most THV technology (radial force) and the proximity of the implantation site to the cardiac conduction system. That is, even if we had implanted the valve even shallower, the use of larger valves with aggressive oversizing in TAVR for PNAR may eventually still result in higher PPI rates. This theory is supported by real-world data showing that the PPI rate following the use of Evolut-R $34 \mathrm{~mm}$ in patients with annulus $\geq 26 \mathrm{~mm}$ was very high (up to $20 \%$ ) (31).

Although it has been consistently shown that newergeneration THVs in TAVR for PNAR are associated with more device success, one key determinant for the success of newer-generation devices is the development of dedicated TAVR devices for PNAR (2-11). As shown in Yoon et al., among the newer-generation valves implanted, JenaValve (JenaValve, Munich, Germany) was the most used one (30.2\%) (4). The nitinol loops of JenaValve act as anchors around the native aortic valve leaflets and allow the THV to be deployed inside them. The transapical implantation of the JenaValve in the earliest 31 patients with PNAR was successful in 30 of them (97\%), and the all-cause mortality was $19 \%$ at 6 months postoperatively (7). Like the JenaValve, the J-Valve has graspers that fix on the native leaflets that facilitate intuitive self-positioning valve implantation. A successful first-in-human implantation was reported in 2015 , and its early results were also satisfactory: 97.7\% procedural success, $77 \%$ absent or trace PVL, $4.7 \%$ PPI rate, and $4.7 \%$ all-cause mortality at 1 year, were reported (9). Our three patients with compassionate-use indications underwent transapical TAVR with the J-valve in 2020. The procedures were all successful, with no/trace post-procedural PVL in all cases, although one patient needed PPI.

Since the TF approach has been the preferred route for TAVR implantation, both JenaValve and J-valve devices with grasping mechanisms have to be modified and evolved to be placed via peripheral access. The JUPITER registry described the 1-year outcomes with the next-generation TF JenaValve device: $97 \%$ procedural success, $85 \%$ absent or trace PVL, $4 \%$ PPI rate, and $80 \%$ survival at 1 year, were reported (8). Currently, an early feasibility study of the TF JenaValve system is underway in the United States (The ALIGN-AR Trial) (32). Moreover, the first TF J-valve (JC Medical, Burlingame, CA, USA) TAVR system was successfully implanted in-human in 2019 (33) and the company is currently enrolling in a compassionate-use study in $\mathrm{AR}$ patient subsets at a high/prohibitive surgical risk for which there are no alternative treatments (34). In sum, the presently available data demonstrates that these two dedicated TAVR devices for use in PNAR are promising (7-9,33). If the outcomes of ongoing larger clinical trials are positive, they will in turn lead to commercial approval and eventually make TAVR ready for use in $\operatorname{PNAR}(32,34)$.

Finally, in the present study, we found that higher 
baseline STS-PROM score $(\mathrm{P}<0.01)$ and longer intensive care unit stays were independently associated with the primary end-points by multivariate analysis (Table 4). Our findings are consistent with those reported in previous TAVR for PNAR studies, showing that the mid- and longterm mortality may be affected by procedural complications as well as baseline comorbidities (3-5). Moreover, we noticed a trend towards better long-term clinical outcomes with the use of newer-generation THVs by Kaplan-Meir analysis, although this seems to be statistically insignificant. This may be attributable to the small sample size and the poor underlying conditions of the study patients. Hence, further studies on TAVR are needed in this challenging population to optimize the procedural and also the longterm clinical outcomes, before the sequelae of severe PNAR become irreversible.

\section{Study limitations}

The present analysis has to be interpreted in light of several limitations. Firstly, this was not a randomized trial, and is thus subject to selection bias and unmeasured confounders. Secondly, the relatively small sample size did not allow comparison of different THV types, especially the newer-generation self-expanding valves and the dedicated TAVR devices. Therefore, conclusions cannot be drawn from the present study. Thirdly, device selection was not randomized, but rather at the operator's discretion and significantly influenced by their experiences, which may have affected the observed outcomes. Fourthly, the study period was relatively long, spanning a period of more than 5 years. The evolution in patient selection, accumulating operator/center experiences, improvements in transcatheter valve technology, and the advent of newer-generation device iterations may all contribute to the reduction of complication rates and improvement of TAVR results, and make it difficult to estimate the real effect of each factor on the overall TAVR outcomes. Nevertheless, the bottom line is that new technology has addressed the limitations from larger annular sizes secondary to aortopathies, embolization/ migration owing to a lack of calcium, and residual PVL due to difficult placement. Procedural results have been improved with the use of newer-generation devices, which have more size varieties and the ability to be repositioned/ recaptured. The dedicated TAVR devices for PNAR with mechanisms to grasp native leaflets are associated with a significantly higher procedural success rate, and may be so preferred to other newer-generation devices.

\section{Conclusions}

Early-generation TAVR devices are associated with less satisfactory outcomes in the treatment of patients with PNAR and should be avoided. TAVR using newergeneration THVs has yielded better procedural outcomes and can be a great asset to treat certain patients. Dedicated TAVR devices for PNAR are preferred to other newergeneration devices.

\section{Acknowledgments}

We thank Ms. Chin-Feng Cheng for her assistance in preparing this manuscript.

A part of data had previously accepted as an abstract in the 29th Great Wall International Congress of Cardiology and the 2021 Annual Scientific Meeting of the Formosan Medical Association.

Funding: None.

\section{Footnote}

Reporting Checklist: The authors have completed the TREND reporting checklist. Available at https://dx.doi. org/10.21037/atm-21-6936

Data Sharing Statement: Available at https://dx.doi. org/10.21037/atm-21-6936

Conflicts of Interest: All authors have completed the ICMJE uniform disclosure form (available at https://dx.doi. org/10.21037/atm-21-6936). The authors have no conflicts of interest to declare.

Etbical Statement: The authors are accountable for all aspects of the work in ensuring that questions related to the accuracy or integrity of any part of the work are appropriately investigated and resolved. The study was conducted in accordance with the Declaration of Helsinki (as revised in 2013), and was approved by the Institutional Review Board of Cheng Hsin General Hospital [No. (523) 104-59-3]. The requirement for individual consent for this retrospective analysis was waived.

Open Access Statement: This is an Open Access article distributed in accordance with the Creative Commons Attribution-NonCommercial-NoDerivs 4.0 International License (CC BY-NC-ND 4.0), which permits the non- 
commercial replication and distribution of the article with the strict proviso that no changes or edits are made and the original work is properly cited (including links to both the formal publication through the relevant DOI and the license). See: https://creativecommons.org/licenses/by-nc-nd/4.0/.

\section{References}

1. Writing Committee Members; Otto CM, Nishimura RA, et al. 2020 ACC/AHA guideline for the management of patients with valvular heart disease: A report of the American College of Cardiology/American Heart Association Joint Committee on Clinical Practice Guidelines. J Thorac Cardiovasc Surg 2021;162:e183-353.

2. Testa L, Latib A, Rossi ML, et al. CoreValve implantation for severe aortic regurgitation: a multicenter registry. Eurointervention 2014;10:739-45.

3. Sawaya FJ, Deutsch MA, Seiffert M, et al. Safety and Efficacy of Transcatheter Aortic Valve Replacement in the Treatment of Pure Aortic Regurgitation in Native Valves and Failing Surgical Bioprostheses: Results From an International Registry Study. JACC Cardiovasc Interv 2017;10:1048-56.

4. Yoon SH, Schmidt T, Bleiziffer S, et al. Transcatheter Aortic Valve Replacement in Pure Native Aortic Valve Regurgitation. J Am Coll Cardiol 2017;70:2752-63.

5. Anwaruddin S, Desai ND, Szeto WY, et al. Self-Expanding Valve System for Treatment of Native Aortic Regurgitation by Transcatheter Aortic Valve Implantation (from the STS/ACC TVT Registry). Am J Cardiol 2019;124:781-8.

6. De Backer O, Pilgrim T, Simonato $M$, et al. Usefulness of Transcatheter Aortic Valve Implantation for Treatment of Pure Native Aortic Valve Regurgitation. Am J Cardiol 2018;122:1028-35.

7. Seiffert M, Bader R, Kappert U, et al. Initial German experience with transapical implantation of a secondgeneration transcatheter heart valve for the treatment of aortic regurgitation. JACC Cardiovasc Interv 2014;7:1168-74.

8. Silaschi M, Conradi L, Wendler O, et al. The JUPITER registry: One-year outcomes of transapical aortic valve implantation using a second generation transcatheter heart valve for aortic regurgitation. Catheter Cardiovasc Interv 2018;91:1345-51.

9. Liu H, Yang Y, Wang W, et al. Transapical transcatheter aortic valve replacement for aortic regurgitation with a second-generation heart valve. J Thorac Cardiovasc Surg 2018;156:106-16.
10. Rawasia WF, Khan MS, Usman MS, et al. Safety and efficacy of transcatheter aortic valve replacement for native aortic valve regurgitation: A systematic review and metaanalysis. Catheter Cardiovasc Interv 2019;93:345-53.

11. Wernly B, Eder S, Navarese EP, et al. Transcatheter aortic valve replacement for pure aortic valve regurgitation: "onlabel" versus "off-label" use of TAVR devices. Clin Res Cardiol 2019;108:921-30.

12. Iung B, Baron $G$, Butchart EG, et al. A prospective survey of patients with valvular heart disease in Europe: The Euro Heart Survey on Valvular Heart Disease. Eur Heart J 2003;24:1231-43.

13. Brzezinski A, Koprivanac M, Gillinov AM, Mihaljevic T. Pathophysiology of aortic valve disease. In: Cohn LH, Adams DH, eds. Cardiac Surgery in the Adult. New York (New York): McGraw-Hill Education/Medical, 2018.

14. de Meester C, Gerber BL, Vancraeynest D, et al. Do Guideline-Based Indications Result in an Outcome Penalty for Patients With Severe Aortic Regurgitation? JACC Cardiovasc Imaging 2019;12:2126-38.

15. Zoghbi WA, Chambers JB, Dumesnil JG, et al. Recommendations for evaluation of prosthetic valves with echocardiography and doppler ultrasound: a report From the American Society of Echocardiography's Guidelines and Standards Committee and the Task Force on Prosthetic Valves, developed in conjunction with the American College of Cardiology Cardiovascular Imaging Committee, Cardiac Imaging Committee of the American Heart Association, the European Association of Echocardiography, a registered branch of the European Society of Cardiology, the Japanese Society of Echocardiography and the Canadian Society of Echocardiography, endorsed by the American College of Cardiology Foundation, American Heart Association, European Association of Echocardiography, a registered branch of the European Society of Cardiology, the Japanese Society of Echocardiography, and Canadian Society of Echocardiography. J Am Soc Echocardiogr 2009;22:975-1014; quiz 1082-4.

16. Kappetein AP, Head SJ, Généreux P, et al. Updated standardized endpoint definitions for transcatheter aortic valve implantation: the Valve Academic Research Consortium-2 consensus document. Eur Heart J 2012;33:2403-18.

17. Zhu D, Chen W, Peng L, et al. Valve sizing for pure aortic regurgitation during transcatheter aortic valve replacement: deformation dynamic of the aortic annulus in different valve pathology may be different. JACC Cardiovasc Interv 
2015;8:372-3.

18. Markham R, Ghodsian M, Sharma R. TAVR in Patients with Pure Aortic Regurgitation: Ready to Use? Curr Cardiol Rep 2020;22:98.

19. Latib A, Michev I, Laborde JC, et al. Post-implantation repositioning of the CoreValve percutaneous aortic valve. JACC Cardiovasc Interv 2010;3:119-21.

20. Waterbury TM, Reeder GS, Pislaru SV, et al. Techniques and outcomes of paravalvular leak repair after transcatheter aortic valve replacement. Catheter Cardiovasc Interv 2017;90:870-7.

21. Guérios ÊE, Gloekler S, Pilgrim T, et al. Second valve implantation for the treatment of a malpositioned transcatheter aortic valve. J Invasive Cardiol 2012;24:457-62.

22. Wilson W, Osten M, Horlick E. Edwards Sapien XT valve implantation in CoreValve aortic prosthesis to treat severe paravalvular regurgitation. Int J Cardiol 2014;174:e105-7.

23. Taramasso M, Sharp AS, Maisano F. First-in-man case report of the use of an Edwards-Sapien valve to treat a regurgitant CoreValve aortic valve prosthesis. Catheter Cardiovasc Interv 2010;75:51-5.

24. Buzzatti N, Montorfano M, Romano V, et al. A computed tomography study of coronary access and coronary obstruction after redo transcatheter aortic valve implantation. EuroIntervention 2020;16:e1005-13.

25. Gomes B, Geis NA, Chorianopoulos E, et al. Improvements of Procedural Results With a NewGeneration Self-Expanding Transfemoral Aortic Valve Prosthesis in Comparison to the Old-Generation Device. J Interv Cardiol 2017;30:72-8.

26. De Torres-Alba F, Kaleschke G, Diller GP, et al. Changes in the Pacemaker Rate After Transition From Edwards SAPIEN XT to SAPIEN 3 Transcatheter Aortic Valve Implantation: The Critical Role of Valve Implantation Height. JACC Cardiovasc Interv 2016;9:805-13.

27. Jilaihawi H, Zhao Z, Du R, et al. Minimizing Permanent

Cite this article as: Yin WH, Lee YT, Tsao TP, Lee KC, Hsiung MC, Wei J. Outcomes of transcatheter aortic valve replacement for pure native aortic regurgitation with the use of newer- $v s$. early-generation devices. Ann Transl Med 2022;10(1):24. doi: 10.21037/atm-21-6936
Pacemaker Following Repositionable Self-Expanding Transcatheter Aortic Valve Replacement. JACC Cardiovasc Interv 2019;12:1796-807.

28. Tang GHL, Zaid S, Michev I, et al. "Cusp-Overlap" View Simplifies Fluoroscopy-Guided Implantation of Self-Expanding Valve in Transcatheter Aortic Valve Replacement. JACC Cardiovasc Interv 2018;11:1663-5.

29. Gada H, Vora A, Siddique S, et al. TCT CONNECT-457 Reduction of rates of permanent pacemaker implantation with 34-mm Evolut R using cusp overlap technique. J Am Coll Cardiol 2020;76:B196.

30. Maeno Y, Yoon SH, Abramowitz Y, et al. Effect of ascending aortic dimension on acute procedural success following self-expanding transcatheter aortic valve replacement: A multicenter retrospective analysis. Int J Cardiol 2017;244:100-5.

31. Eitan A, Witt J, Stripling J, et al. Performance of the Evolut-R 34 mm versus Sapien-3 29 mm in Transcatheter aortic valve replacement patients with larger annuli: Early outcome results of Evolut-R $34 \mathrm{~mm}$ as compared with Sapien-3 $29 \mathrm{~mm}$ in patients with Annuli $\geq 26 \mathrm{~mm}$. Catheter Cardiovasc Interv 2018;92:1374-9.

32. ClinicalTrials.gov. THE ALIGN-AR TRIAL: JenaValve Pericardial TAVR Aortic Regurgitation Study. Accessed July 14, 2021. Available online: https://clinicaltrials.gov/ ct2/show/NCT02732704

33. Hensey M, Murdoch DJ, Sathananthan J, et al. Firstin-human experience of a new-generation transfemoral transcatheter aortic valve for the treatment of severe aortic regurgitation: the J-Valve transfemoral system. EuroIntervention 2019;14:e1553-5.

34. ClinicalTrials.gov. J-valve compassionate use. Accessed July 14, 2021. Available online: https://clinicaltrials.gov/ ct2/show/NCT03876964

(English Language Editor: A. Kassem) 